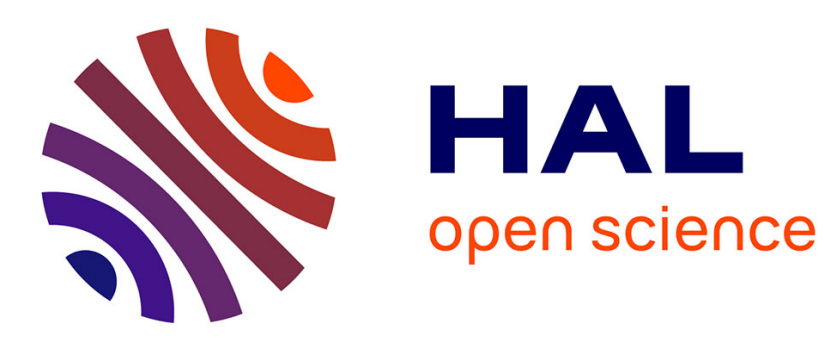

\title{
Ultrafast Melting of Metal-Organic Frameworks for Advanced Nanophotonics
}

Nikita Kulachenkov, Stéphanie Bruyere, Sergey Sapchenko, Yuri Mezenov, Dapeng Sun, Andrei Krasilin, Alexandre Nominé, Jaâfar Ghanbaja, Thierry Belmonte, Vladimir Fedin, et al.

\section{To cite this version:}

Nikita Kulachenkov, Stéphanie Bruyere, Sergey Sapchenko, Yuri Mezenov, Dapeng Sun, et al.. Ultrafast Melting of Metal-Organic Frameworks for Advanced Nanophotonics. Advanced Functional Materials, 2020, 30 (7), pp.1908292. 10.1002/adfm.201908292 . hal-02988373

\section{HAL Id: hal-02988373 https://hal.science/hal-02988373}

Submitted on 9 Nov 2020

HAL is a multi-disciplinary open access archive for the deposit and dissemination of scientific research documents, whether they are published or not. The documents may come from teaching and research institutions in France or abroad, or from public or private research centers.
L'archive ouverte pluridisciplinaire HAL, est destinée au dépôt et à la diffusion de documents scientifiques de niveau recherche, publiés ou non, émanant des établissements d'enseignement et de recherche français ou étrangers, des laboratoires publics ou privés. 


\section{Ultrafast Melting of Metal-Organic Frameworks for Advanced Nanophotonics}

Nikita K. Kulachenkov ${ }^{1}$, Stéphanie Bruyere ${ }^{2}$, Sergey A. Sapchenko ${ }^{3,4,5}$, Yuri A. Mezenov ${ }^{1}$, Dapeng Sun $^{6}$, Andrei A. Krasilin ${ }^{1,7}$, Alexandre Nominé ${ }^{1,2}$, Jaâfar Ghanbaja ${ }^{2}$, Thierry Belmonte ${ }^{2}$, Vladimir P. Fedin, Evgeny A. Pidko ${ }^{6,8}$, and Valentin A. Milichko ${ }^{1}$

${ }^{1}$ Faculty of Physics and Engineering ITMO University St. Petersburg 197101, Russia

${ }^{2}$ Universite de Lorraine CNRS, IJL F-54000 Nancy, France

${ }^{3}$ School of Chemistry University of Manchester Oxford Road, Manchester M13 9PL, UK

${ }^{4}$ Institute of Inorganic Chemistry SB RAS Novosibirsk 630090, Russia

${ }^{5}$ Faculty of Natural Sciences Novosibirsk State University Novosibirsk 630090, Russia

${ }^{6}$ Inorganic Systems Engineering Group Department of Chemical Engineering Delft University of Technology 2629 HZ, Delft, The Netherlands

${ }^{7}$ Ioffe Institute St. Petersburg 197101, Russia

${ }^{8}$ TheoMAT Group ChemBio Cluster ITMO University Saint Petersburg 191002, Russia 


\begin{abstract}
The conversion of metal-organic frameworks (MOFs) into derivatives with a well-defined shape and composition is considered a reliable way to produce efficient catalysts and energy capacitors at the nanometer scale. Yet, approaches based on conventional melting of MOFs provide the derivatives such as amorphous carbon, metal oxides, or metallic nanoclusters with an appropriate morphology. Here ultrafast melting of MOFs is utilized by femtosecond laser pulses to produce a new generation of derivatives with complex morphology and enhanced nonlinear optical response. It is revealed that such a nonequilibrium process allows conversion of interpenetrated 3D MOFs comprising flexible ligands into well-organized spheres with a metal oxide dendrite core and amorphous organic shell. The ability to produce such derivatives with a complex morphology is directly dependent on the electronic structure, crystal density, ligand flexibility, and morphology of initial MOFs. An enhanced second harmonic generation and three-photon luminescence are also demonstrated due to the resonant interaction of 100-1000 nm spherical derivatives with light. The results obtained are in the favor of new approaches for melting special types of MOFs for nonlinear nanophotonics.
\end{abstract}




\section{Introduction}

Metal-organic frameworks (MOFs) as novel class of molecular crystals stand out by their unconventional hierarchical structure based on weak and strong interactions between various building blocks such as organic ligands, metal nodes, and solvent molecules. Their co-operation causes MOF compounds to possess an ordered porous structure[1] and softness[2] making them to be perspective materials for many applications in chemistry,[3] biology,[4] and physics in general.[5] Besides this unique combination of properties, one of the most intriguing features of MOFs is the dynamical response of their structure on external stimuli such as pressure, temperature, electric field, and even light.[6] This opens up an ability for efficient utilizing of the MOFs for data storage,[5b-d] controlled filtering,[6c] and nonlinear mechanics.[5e] Meanwhile, under extremal conditions, such stimuli allow the obtaining of new stable structures such as nanometer scale derivatives,[7] which turned out to be very useful for energy storage[7b-d] and catalysis[7e] due to their morphology and nanometer sizes.

The production of functional MOF derivatives, such as amorphous carbon, metal oxides, chalcogenides, phosphides, carbides, or pure metallic nanoclusters, [7a-c] has become one of the most rapidly developing field of nanoscience. However, the approaches to fabricate them are generally based on relatively slow processes such as heating to the melting point within a broad time ranging from nanoseconds to hours. Important is that, these processes limit the synthesis product to the stable structures. In contrast, implementation of nonequilibrium approach of injecting high energy density over a shorter timespan (up to femtoseconds) enables the formation of metastable structures, which are completely new in terms of morphology. Indeed, nonequilibrium processes possess ultrafast melting and cooling rates with complicated dynamics of solidification resulting in dramatic impact on the final morphology and properties of the nanometer scale structures.[8a-d] As a result, this significantly widens the library of nanomaterials and their application in nanophotonics making them competitive with existing nonlinear optical nanomaterials as inorganic nanoparticles[8d] and organic/ inorganic 2D materials.[8e-i] Therefore, research on the effective ways of MOFs conversion to a new kind of derivatives employing ultrafast melting seems to be a substantial step toward synthesis of new nanomaterials with unique functionality.

Here we utilize for the first time an ultrafast melting of MOFs with flexible alkane-chained ligands (Figure 1a) and interpenetrated 3D structure (Figure 2) by femtosecond (fs) IR laser pulses. This allows us to investigate light-matter interactions with regard to the production of new forms of MOF derivatives for nonlinear nanophotonics. We reveal the interplay between the initial MOF structure and the morphology and composition of the derivatives, as well as their nonlinear optical properties. Overall, the proposed approach for ultrafast melting of MOFs leads to conceptually new nanometerscale derivatives and opens up new perspectives for an efficient postsynthetic processing of MOF compounds to yield a family of materials with a rarely described combination of nonlinear optics, and potentially catalytic and energy storage application.

\section{Results and Discussion}

As mentioned above, classical approaches to melt MOFs for producing different nanometer scale derivatives,[7a-c] amorphous phases,[9a-e] and MOF glasses[10] (Figure S1, Supporting Information) take much longer time ranging from tens of nanoseconds[11] to hours[10] which is sufficient for reaching the thermal equilibrium. In contrast, under the strong femtosecond optical excitation,[12a] MOFs like other crystalline semiconductors (bismuth,[12b] silicon,[12c] indium antimonide,[12d,e] germanium,[12f ] and gallium arsenide[12g]) should undergo the transition to the state at which few percent of electrons exited from valence to conduction band induce changes in the potential-energy surface and temporary modification of the crystal lattice (Figure 1b). They drastically affect the process of light-induced melting of semiconductors. 
The fact that fs melting occurs before the thermal equilibrium (nonthermal melting) between electrons and the lattice through electron-phonon interaction[12] raises the question of the possibility to convert MOFs into new metastable states with new functionality.

\subsection{Model MOFs}

\subsubsection{Synthesis}

As a model system, we use the series of 3D MOFs (1 to 3) previously synthetized by Barsukova et al.[13] All of them contain 4,4'-stilbenedicraboxylate $\left(\mathrm{sdc}^{2-}\right)$ and imidazolyl-based ligands featuring flexible hydrocarbon chains of different lengths (Figure 1a): 1,4-di(1H-imidazol-1-yl)butane (L4) or 1,6- di(1H-imidazol-1-yl)hexane (L6). Compound 1 crystallizes in monoclinic space group $P 2 / c$ and has quite dense and rigid structure with chemical formula $[\mathrm{Cd}(\mathrm{sdc})(\mathbf{L 4})] \cdot \mathrm{DMF}$ (DMF, dimethylformamide). It comprises sixfold interpenetrated frameworks of a diamond topology (dia). Compound 2 also contains cadmium (II) but has a larger imidazolyl-based ligand L6. Its chemical composition is $[\mathrm{Cd}(\mathrm{dmf})(\mathrm{sdc})(\mathrm{L} 6)] \cdot \mathrm{DMF}$. From structural point of view, the compound is characterized by a very high interpenetration degree (eightfold) of the diamond-type frameworks built from cadmium cations connected through $\mathrm{sdc}^{2-}$ and L6 ligands. Importantly, it crystallizes in a noncentrosymmetric space group $P 2_{1} 2_{1} 2_{1}$. Compound 3 is zinc(II)-based and its formula is $[\mathrm{Zn}(\mathrm{sdc})(\mathbf{L 6})] \cdot \mathrm{DMF}$. Its structure also demonstrates eightfold interpenetration of diamond-type frameworks, but it has highest free accessible volume of $\approx 21 \%$ among all three compounds and the smallest crystallographic density of $1.228 \mathrm{~g} \mathrm{~cm}^{-3}$ as opposed to $1.493 \mathrm{~g} \mathrm{~cm}^{-3}$ for 1 and $1.387 \mathrm{~g} \mathrm{~cm}^{-3}$ for 2. It should be noted that MOFs with such complex structures have not been investigated recently as a starting compounds for a postsynthetic conversion. However, despite the intrinsic complexity of these compounds we chose them as starting materials for the experiments expecting some of their structural features (high interpenetration degree and presence of weakly interacting flexible ligands) would play a role in the formation of molten species with new morphology, different from other wellknown MOF derivatives.

\subsubsection{Characterization}

To perform the ultrafast melting together with optical characterization, we utilize a self-made setup comprising an Yb-doped solid-state femtosecond oscillator (150 fs, $1050 \mathrm{~nm}, 80 \mathrm{MHz}$ repetition rate) and aligned objectives for laser focusing with simultaneous optical analysis of initial MOF single crystals and nanometer scale objects by commercial photo camera and confocal spectrometer HORIBA LabRam (Figure S2, Supporting Information).

The MOF samples used in this work were obtained solvothermally in accordance with published procedure.[13] The optical measurements allowed us to estimate the bandgap for compounds 1-3 $(\approx 2.9 \mathrm{eV})[13]$ and revealed high transparency within the visible and near IR range (Figure S3, Supporting Information), which makes them stable to IR irradiation. Moreover, every MOF samples being investigated demonstrate different nonlinear optical effects (Figure 2):

a) Single crystals of 1,2, and 3 demonstrate pronounced nonlinear optical effect such as three-photon luminescence (3PL). In this case, the energy of photons, $\mathrm{E}_{3 \mathrm{PL}}$, noncoherently emitted by the crystals, is equal to $\mathrm{E}_{3 \mathrm{PL}}=3 * \mathrm{E}_{\mathrm{ph}}-\mathrm{E}_{\mathrm{nr}}$, where $\mathrm{E}_{\mathrm{ph}}$ is an energy of IR photons $(1.18 \mathrm{eV})$ and $\mathrm{E}_{\mathrm{nr}}$ corresponds to the energy of nonradiative processes. In case of 1, the 3PL spectrum covers the range from 2.2 to $3.1 \mathrm{eV}$ with corresponding cubic slope $2.7 \pm 0.2$ on excitation intensity (Figure 2a). 3PL signal with the same spectral range and the slope of $3.0 \pm 0.1$ is also observed for compound 2 (Figure $2 b$ ). It should be noted that the most intensive signal of 3PL is observed for this compound (Figure S4, Supporting Information). Regarding compound 3, 3PL can be observed in more specific form (Figure 2c): photoluminescence is characterized by changing shape of spectra with lower slope $(2.5 \pm 0.3)$ and strong dependence on the fluence (Figure S5, Supporting Information). 
b) Intriguing for all compounds we confirm another nonlinear optical effect as second harmonic generation (SHG), i.e., coherently emitting doubled frequencies $\left(1 / \lambda_{1}+1 / \lambda_{1}=1 / \lambda_{2}\right)$, where $\lambda_{1}$ and $\lambda_{2}$ are the wavelengths of excitation $(1050 \mathrm{~nm})$ and emitted photons $(525 \mathrm{~nm})$. This quadratic optical nonlinearity is usually observed for materials having non-centrosymmetric space groups; however, SHG can be also detected for some semiconductors due to surface effects or light-induced breaking symmetry.[12a,14a-c] Concerning compound 1, which has centrosymmetric space group P2c, we detect SHG with quadratic slope of $1.85 \pm 0.15$ which is appeared under the fluence of $\approx 0.7-7.5 \mathrm{~mJ}$ $\mathrm{cm}^{-2}$ (Figure 2a). The lower limit may be caused by a threshold energy needed for breaking the symmetry,[12a] while the upper limit corresponds to the state when the slop for SHG deviates from quadratic to linear function on the fluence. This behavior can be also explained by sufficient lightinduced structural changes[12a] of compound 1 having weakly interacting flexible alkali-chained ligands. In opposite to 1 , single crystals of 2 have non-centrosymmetric space group $P 2{ }_{l}{ }_{2} 2_{l}$ and also generate second harmonic radiation at a wavelength of $525 \mathrm{~nm}$ with quadratic slope of $1.8 \pm 0.2$ (Figure 2b). The SHG signal is detected under the broad fluence range from 0.2 to $6 \mathrm{~mJ} \mathrm{~cm}^{-2}$. The lower limit is due to the sensitivity of spectrometer, while the upper limit may be caused by the lightinduced structural changes as for 1. Similar to 3PL efficiency (Figure S4, Supporting Information), compound 2 also emits SHG with a maximal efficiency. Finally, compound 3 with centrosymmetric space group Pbcn emits SHG in more specific form: the emission is stepwise and observed at higher fluence threshold of $\approx 2 \mathrm{~mJ} \mathrm{~cm}^{-2}$ with the slope of $1.75 \pm 0.15$. At higher fluence of $\approx 5.5 \mathrm{~mJ} \mathrm{~cm}{ }^{-2}$ the signal vanishes. Such deviations of 3PL and SHG signals for 3 can be explained by more dynamic reaction of the compound to the pulsed intense radiation, as its structure comprises the flexible ligands of the maximal length and has the lowest crystal density among all three complexes providing a greater degree of freedom for 3 to change its structure upon irradiation.

\subsection{Ultrafast Melting: An Overview}

Once the preliminary characterization was done, we utilize our setup (Figure S2, Supporting Information) to produce derivatives by exposition of the MOF single crystals to $150 \mathrm{fs}$ IR laser pulses (Figure 3a). By increasing the laser power from 0.2 to $15 \mathrm{~mJ} \mathrm{~cm}-2$ and measuring the MOF response in terms of structure (Raman scattering), intensity of nonlinear optical signals (3PL and SHG) and dark-field optical imaging, we clearly identify four regimes I-IV (Figure 3b). Further, we consider them on the example of compound 1 :

I) A nonperturbed regime (the fluence up to $7.5 \mathrm{~mJ} \mathrm{~cm}^{-2}$ ) corresponds to stable signals of SHG (Figure 3d) and 3PL with quadratic and cubic slopes, respectively. The Raman scattering also confirms nonperturbed structure after irradiation with the fluence less than $7.5 \mathrm{~mJ} \mathrm{~cm}^{-2}$ (Figure 3c).

II) The transition regime (the fluence from 7.5 to $9.5 \mathrm{~mJ} \mathrm{~cm}$ ) is characterized by variation of the intensity of SHG (Figure S5d, Supporting Information) and deviation of the slopes of SHG and 3PL from cubic and quadratic dependence to linear functions, respectively. The following decrease of fluence yields a transition to regime I, but with lower intensity of SHG (3PL) and is accompanied by a slight color changes of the crystals. These can be caused by formation of crystal defects[9f,g] under irradiation and actually makes the transition I to II irreversible in terms of structure.

III) The melting regime is appeared with the threshold transition at $\approx 9.5 \mathrm{~mJ} \mathrm{~cm}^{-2}$. This regime is characterized by a step-like melting of the MOF crystals and possibility to produce the particles, which efficiently scatter the light and, hence, have different colors (Figure 4a). Such specific behavior during the melting is similar to the transition between thermal and ultrafast nonthermal melting of crystalline semiconductors under intense fs laser pulses.[12a-e] This hypothesis is also supported by the fact that we can achieve MOF melting under $9 \mathrm{~mJ} \mathrm{~cm}^{-2}$ with long exposition (more than $10 \mathrm{~min}$ ), while increase of 
fluence by $10 \%$ to $10 \mathrm{~mJ} \mathrm{~cm}^{-2}$ results in the snap melting. This observation can also speak in favor of first nonthermal melting of MOFs.

IV) Finally, for the last regime, the burning of the whole crystals (inset in Figure 3b) with creation of small amount of nanometer scale particles can be observed at higher fluence $\left(>11.5 \mathrm{~mJ} \mathrm{~cm}^{-2}\right)$.

Following the concept of ultrafast melting of semiconductors by exiting few percent of electrons from valence to conduction bands,[12] the fraction of excited electrons for each investigated compound could be roughly estimated as following: the ultrafast melting of 1 occurs under 3-6\% of exited electrons, while $2.5 \%$ and $2.2 \%$ correspond to 2 and 3, respectively (for the details, see Supporting Information). The results are in a good agreement with previously reported data on nonthermal ultrafast laser melting of other crystalline semiconductors (Figure $1 \mathrm{~b}$ and Table S2, Supporting Information). The increase in the number of exited electrons from $2.2 \%$ to $3 \%$ correlates well with the gradual growth of MOFs crystal density from compound 3 to 1 . Moreover, the estimated value of $\approx 3 \%$ for all compounds corresponds well to the values of 3-5\% obtained for other materials with melting points (Bi, M.P. $280{ }^{\circ} \mathrm{C}$ and InSb, M.P. $530{ }^{\circ} \mathrm{C}$ ) close to MOFs used in this study (M.P. at $\approx 400$ $\left.{ }^{\circ} \mathrm{C}\right)$.[13] The crystalline semiconductors with higher melting point $\left(>940{ }^{\circ} \mathrm{C}\right)$ require more than $6 \%$ of exited valence electrons (Figure 1b). We can argue that the presence of number of relatively weak bonds, which are involved in the formation of crystal structurer of MOFs, is also responsible for a decreased value of exited electrons needed to weaken these bonds and soften the MOF lattice.

Statistical investigation of the interaction of 40 single crystals of 1,2, and 3 with fs pulses of different fluence was also performed (Figure $3 \mathrm{~b}$ ). We reveal that compound 1 is more rigid to the increasing fluence than compound 3 , while compound 2 was intermediate in terms of stability. The explanation of this effect (i.e., different fluence thresholds for ultrafast melting) can be given with regard to the analysis of ultrafast dynamics of the interpenetrated structure of such MOFs with flexible building blocks, as well as thorough analysis of metal-nitrogen (metal-oxide) strength.[10a] However, we assume that increase of the crystal density from $\approx 1.2$ to $1.5 \mathrm{~g} \mathrm{~cm}^{-3}$ and decrease in geometrical length of weakly interacting flexible alkali-chained ligands in a row from 3 to 1 limit the compounds' degree of freedom to react on light and their ability to change their conformation. Therefore, the more rigid compound demonstrates higher fluence threshold for melting by fs light. Concerning the thermal stability under the slow heating (see thermogravimetric analysis, TGA, Figure S6, Supporting Information),[13] there is no evidenced link between TGA data and the threshold for ultrafast melting.

\subsection{Ultrafast Melting: The Derivatives}

Since the regime III has been shown to be the most suitable for producing the particles (Figure 3b), we use fluences of 10,8 , and $7 \mathrm{~mJ} \mathrm{~cm}^{-2}$ to produce MOF derivatives from compounds 1,2 , and 3 , respectively (Figure 4a). To carry out both electron microscopy and optical characterization, the derivatives have been printed on carbon grid and gold substrate. Scanning electron microscopy (SEM, FEI Quanta 200) is used to analyze the average shape and size of MOF derivatives. For compound 1 , laser melting mainly provides well-defined spherical particles with diameter distribution from 100 to $1000 \mathrm{~nm}$ (Figure 4b). In contrast, compounds 2 and 3 are characterized by irregular shaped structures with characteristic size from 800 to $3800 \mathrm{~nm}$ and 500 to $2500 \mathrm{~nm}$, respectively, which can be easily destroyed by electron beam of SEM. Transmission electron microscopy (TEM) and energy dispersive X-ray (EDX) with semi-quantitative elemental analysis are also used to provide insights into the structural and composition properties of the derivatives (Figure 5 and Figure S7, Supporting Information). TEM (JEOL ARM 200F TEM/scanning TEM operating at $200 \mathrm{kV}$ ) reveals that derivatives have completely different morphology. In case of 1, two morphologically different types of particles were produced: the amorphized drops (Figure 5k) with metal-organic composition (Figure $51, \mathrm{~m}$ and Table S3, Supporting Information) and homogeneous redistribution of the elements (Figure $5 \mathrm{n}$ ); and spherical ones (Figure $5 \mathrm{a}-\mathrm{c}, \mathrm{g}-\mathrm{j}$ ) whose internal structure can be described as core-shell (Figure 5f). The shell represents an amorphized organic phase, which is confirmed by elemental 
analysis (Figure 5d,e and Table S3, Supporting Information), Raman scattering (Figure 3c), and redshifted 3PL spectra (Figure 6a). Surprisingly, the absence of carbon contamination is verified by Raman scattering, which makes the obtained derivatives to be very different from the reported recently. In the latter case, amorphous carbon becomes a regular product. In contrast, the core of the derivatives has the more complicated morphology as dendrite-like metal-oxide confirmed by TEM and EDX analysis (Figure 5c-j). To the best of our knowledge, such unique core-shell structures have not been previously synthesized from MOFs.

In case of 2 and 3, we produce only the derivatives of irregular shape (Figure S7a,d, Supporting Information) and amorphous phase with metal-organic composition (Figure S7b,c,g,h and Table S3, Supporting Information) confirmed by TEM and redshifted 3PL (Figure 6c). It should be noted that elemental analysis reveals the differences between composition of initial MOFs and all their derivatives (Table S3, Supporting Information), which is in good agreement with results on MOF melting by classical ways.[10a] The redshifted 3PL signal also confirms the presence of amorphous organic phase, while the existence of cadmium and zinc atoms and their redistribution over the shape of the derivatives is attested by EDX analysis (Figure S7c,h,i, Supporting Information).

To shed light on the reasons for the fundamental difference in the morphology of the derivatives obtained under the ultrafast optical excitation, we analyze the electronic structure of the MOFs by means of density functional theory (DFT) calculations. Figure S9 in the Supporting Information presents the computed projected density of states (pDOS) for 1,2 , and 3 and the respective banddecomposed partial charge densities at the valance band maximum (VBM) and the conduction band minimum (CBM). Calculations underestimate the bandgaps $(2.1,2.0$, and $2.2 \mathrm{eV}$, respectively) for 1 , 2 , and 3 compared to the experimental values $(\approx 2.9 \mathrm{eV})$ due to the known shortcomings of the generalized gradient approximatio-type functionals.[14d,e] The results in Figure S9 in the Supporting Information reveal substantial differences in the electronic structure of 1 compared to that of 2 and 3 . DFT calculations show that for compound 1 the VBM is mainly represented by the $\mathrm{C}-\mathrm{C}$ bonding $\pi$ orbitals and the $\mathrm{O} 2 \mathrm{p}$ orbitals. Substantial contribution of Cd orbitals to the valence band close to the Fermi level could also be noted. The corresponding electronic charge densities of VBM show a pronounced delocalization among $\mathrm{Cd}, \mathrm{O}$, and the aromatic $\pi$-system. The CBM is mainly represented by the $\pi^{*}$ systems of the ligands with some contributions due to $\mathrm{O}$ atoms of the carboxylate moieties. Both VBM and CBM are delocalized along the structure-forming Cdcarboxylate chains and span throughout the crystal. On contrary, for compounds 2 and 3, the delocalization of the VBM and CBM is limited to defined ligand units. The corresponding electronic charge densities are predominantly contributed by the $\pi$ and $\pi^{*}$ aromatic systems of the ligands with only a minor contribution of the orbitals at $\mathrm{O}$ sites. In other words, these differences in the electronic structures suggest that upon optical excitation, 1 would undergo a controlled dissociation in the directions determined by the antibonding character of the delocalized CBM. In the cases of compounds 2 and 3, one cannot expect a specific directionality of the crystal melting upon excitation.

Overall, analyzing the gathered experimental and theoretical information, an important assumption can be made. The interaction of MOFs, having an interpenetrated 3D structure and flexible ligands, with fs laser radiation suggests a dependency between thermal stability, crystal density, and electronic structure of the initial MOFs and the morphology of corresponding derivatives. Indeed, among all three analyzed compounds, complex 1 is less stable at low heating rates (TGA, Figure S6, Supporting Information),[13] has higher crystallographic density, and delocalized electronic density. Perhaps these conditions make it easier to break the bonds in the MOF before it gets completely burned, followed by reorientation and reassembly of MOF structural elements into new structures under nonequilibrium conditions. As a result, only this compound can be converted to core-shell metalorganic derivatives, as opposed to compounds 2 and 3, which are more stable to slow heating and less dense. Their derivatives demonstrate a decreased 3PL and SHG signals (see below) and represent the more homogenous metal-organic structures with much higher degree of amorphization to be formed 
during the ultrafast melting. These structures also cannot be simply considered as amorphous $\operatorname{MOFs}[9 \mathrm{a}-\mathrm{e}, 10]$ as the elemental composition of the resulting derivatives differs significantly from the chemical composition of the starting materials (Table S3, Supporting Information). Furthermore, the presence of coordination bonds in each nanometer scale derivatives appeared challenging to be confirmed by existing techniques.[9e]

\subsection{Application}

Here we consider the dependence of the functionality of the derivatives, obtained from MOFs with intrinsic nonlinear optical properties (Figure 2), on their morphology. In particular, the derivatives obtained especially from 1 have three key features to be considered for application in nonlinear nanophotonics: metal-organic composition, which is important for efficient photoluminescence and enhanced optical response; nonlinear optical response of initial MOFs; and morphology (core-shell structure, size, and shape) which can drive the interaction of light with wavelengths from ultraviolet to IR range.[7f,15] Therefore, we investigate the interaction of light with single derivatives by linear and nonlinear optical microspectroscopy (Figure 6). Concerning nonlinear optical response, we reveal that 3PL spectra of the derivatives obtained from compounds 1 and 2 are redshifted in comparison to that of initial MOFs (Figure 6a,c). This can be explained by amorphized state of the organic components.[16] In contrast, derivatives from 3 do not demonstrate any photoluminescence signal that can be explained either by low signal of 3PL or complete destruction of organic components. The signals of SHG are also preserved for derivatives from 1 and 2 due to the well-developed surface,[15d] distorted organic-inorganic structure,[9a] or light-induced broken symmetry.[9a,14a-c].

It is worth comparing the efficiency of SHG from the nanometer scale derivatives obtained from centrosymmetric crystal 1 with that of nanoparticles with $(\mathrm{Si})$ and without (BaTiO3) centrosymmetric space group (Figure S10, Supporting Information). Interestingly, we detect SHG signal from the derivatives, that is just 2 orders of magnitude smaller than that for BaTiO3 nanoparticles[15f ] of the same diameter $(430 \mathrm{~nm})$. Compared to centrosymmetric and optically resonant $\mathrm{Si}$ nanoparticle with the diameter of $275 \mathrm{~nm}$, for which the well-developed surface of the nanoparticle can be the reason of optical nonlinearity,[15d] MOF derivatives demonstrate the efficiency of the same order of magnitude. The smaller or bigger Si nanoparticles generate second harmonics with efficiency 1 order of magnitude less (Figure S10, Supporting Information).

Moreover, the ratio of intensities of SHG and 3PL for derivatives and corresponding MOFs (1) is different: SHGderiv/3P Lderiv $\approx 5 *$ SHGMOF/3PLMOF. This effect can be explained by resonant interaction of spherical derivatives of diameter from 100 to $1000 \mathrm{~nm}$ and typical refractive index of 2.5 (generally for organic-inorganic materials) with light: high-order Mie-type electromagnetic modes[15] with high quality factor $\mathrm{Q}(\mathrm{Q}=\lambda 0 / \Delta \lambda$, where $\lambda 0$ is the central wavelength of emission and $\Delta \lambda$ is the full width at half maxima) can be optically generated inside such kind of particles supporting an enhancement of intrinsic optical emission.[15d] We reveal these modes by optical spectroscopy (Figure 6b) from single derivatives in the dark-field geometry (Figure S2, Supporting Information). Indeed, SHG signal with high quality $(\mathrm{Q}=138)$ from derivatives overlaps with high order and same high quality Mie modes, [15c-e] which significantly enhance SHG. Concerning 3PL, this broad spectrum with low quality $(\mathrm{Q}=4)$ cannot be significantly affected by the same Mie modes. This situation is for derivatives from 2, where the low quality of Mie-type modes cannot contribute to enhancement of nonlinear optical response.

Based on these results, one can conclude that the more complicated the morphology of MOF derivatives we have (Figure 5c,g,i), the more efficient linear and nonlinear optical response can be observed. This also raises a question of potential application of amorphous MOFs and the derivatives in photonics. Indeed, recent reports of MOF derivatives application were focused on catalysis and energy storage. In this regard, our study is one of the first demonstration of developed optical synthesis of active nanophotonics components based on metal-organic frameworks. 


\section{Conclusion}

For the first time the ultrafast melting of 3D interpenetrated MOFs with flexible alkali-chained ligands by femtosecond IR laser pulses was demonstrated as an effective way to produce unique nanometer scale derivatives with complex morphology and enhanced nonlinear optical response. Structurally, the products of this nonequilibrium process can be categorized as complex derivatives representing wellorganized spheres with metal-oxide dendrite core and amorphous organic (noncarbon) shell or as amorphous metal-organic species. An ability to produce such derivatives and the complexity of their morphology are a result of a tradeoff between the crystal density, ligand length and flexibility, electronic structure, and thermal stability of the starting MOF compounds. The analysis of ultrafast melting also reveals that the denser structure with shorter flexible ligand, the higher the fluence threshold for ultrafast melting of such MOFs. Moreover, the delocalization of electronic density contributes to the process of laserinduced melting and results to complexity of the morphology of MOF derivatives. We also demonstrated an enhanced second harmonic generation and three-photon luminescence due to resonant interaction of the 100-1000 nm spherical derivatives with light. The presented data clearly indicate a vast potential of ultrafast melting of MOF for producing new generation of materials for applications in nonlinear nanophotonics and highlight a general methodology for the research on ultrafast melting of other metal-organic framework complexes and related porous materials.

\section{Supporting Information}

Supporting Information is available from the Wiley Online Library or from the author.

\section{Acknowledgements}

N.K.K. and S.B. contributed equally to this work. Authors thank M. O. Barsukova for providing the samples of MOF compounds for the research. V.A.M., N.K.K., and Y.A.M. acknowledge support from the Russian Foundation for Basic Research (Project No. 18-32-20089 mol_a_ved) for optical investigation of MOF derivatives and the Russian Science Foundation (Projects No. 19-79-10241 and 17-19-01637) for investigation the ultrafast melting of MOFs and their composition, respectively. E.P. acknowledges support from the Ministry of education and Science of the Russian Federation (Project 11.1706.2017/4.6). D.S. and E.P. thank NWO (Nederlandse Organisatie voor Wetenschappelijk Onderzoek) for providing for providing access to the SurfSARA supercomputer facilities. S.B., J.G., and T.B. benefited from Programme Hubert Curien-Kolmogorov and would like to thank Dr.-Ing. P. Boulet (Institut Jean Lamour, CC X-Gamma) for XRD measurements. A.N. acknowledges financial support by the Government of the Russian Federation through the ITMO Fellowship and Professorship program. 


\section{BIBLIOGRAPHY}

[1] a) O. K. Farha, I. Eryazici, N. C. Jeong, B. G. Hauser, C. E. Wilmer, A. A. Sarjeant, R. Q. Snurr, S. B. T. Nguyen, A. Ö. Yazaydın, J. T. Hupp, J. Am. Chem. Soc. 2012, 134, 15016; b) I. M. Hönicke, I. Senkovska, V. Bon, I. A. Baburin, N. Bönisch, S. Raschke, J. D. Evans, S. Kaskel, Angew. Chem. 2018, 130, 13976; c) I. M. Hönicke, I. Senkovska, V. Bon, I. A. Baburin, N. Bönisch, S. Raschke, J. D. Evans, S. Kaskel, Angew. Chem., Int. Ed. 2018, 57, 13780.

[2] a) S. Horike, S. Shimomura, S. Kitagawa, Nat. Chem. 2009, 1, 695; b) A. U. Ortiz, A. Boutin, A. H. Fuchs, F.-X. Coudert, Phys. Rev. Lett. 2012, 109, 195502; c) L. Vanduyfhuys, S. M. J. Rogge, J. Wieme, S. Vandenbrande, G. Maurin, M. Waroquier, V. Van Speybroeck, Nat. Commun. 2018, 9, 204.

[3] a) H. Furukawa, K. E. Cordova, M. O'Keeffe, O. M. Yaghi, Science 2013, 341, 1230444; b) H. Furukawa, U. Müller, O. M. Yaghi, Angew. Chem. 2015, 127, 3480; c) H. Furukawa, U. Müller, O. M. Yaghi, Angew. Chem., Int. Ed. 2015, 54, 3417.

[4] a) K. Lu, T. Aung, N. Guo, R. Weichselbaum, W. Lin, Adv. Mater. 2018, 30, 1707634; b) A. C. McKinlay, R. E. Morris, P. Horcajada, G. Férey, R. Gref, P. Couvreur, C. Serre, Angew. Chem. 2010, 122, 6400; c) A. C. McKinlay, R. E. Morris, P. Horcajada, G. Férey, R. Gref, P. Couvreur, C. Serre, Angew. Chem., Int. Ed. 2010, 49, 6260; d) D. Chen, D. Yang, C. A. Dougherty, W. Lu, H. Wu, X. He, T. Cai, M. E. Van Dort, B. D. Ross, H. Hong, ACS Nano 2017, 11, 4315.

[5] a) Y. A. Mezenov, A. A. Krasilin, V. P. Dzyuba, A. Nominé, V. A. Milichko, Adv. Sci. 2019,6, 1900506; b) S. M. Yoon, S. C. Warren, B. A. Grzybowski, Angew. Chem. 2014, 126, 4526; c) S. M. Yoon, S. C. Warren, B. A. Grzybowski, Angew. Chem., Int. Ed. 2014, 53, 4437; d) G. Wu, J. Huang, Y. Zang, J. He, G. Xu, J. Am. Chem. Soc. 2017, 139, 1360; e) J. M. Zadrozny, A. T. Gallagher, T. D. Harris, D. E. Freedman, J. Am. Chem. Soc. 2017, 139, 7089; f) F. X. Coudert, J. D. Evans, Coord. Chem. Rev. 2019, 388, 48.

[6] a) F. X. Coudert, Chem. Mater. 2015, 27, 1905; b) O. Sato, Nat. Chem. 2016, 8, 644; c) A. Knebel, B. Geppert, K. Volgmann, D. I. Kolokolov, A. G. Stepanov, J. Twiefel, P. Heitjans, D. Volkmer, J. Caro, Science 2017, 358, 347; d) A. P. Katsoulidis, D. Antypov, G. F. S. Whitehead, E. J. Carrington, D. J. Adams, N. G. Berry, G. R. Darling, M. S. Dyer, M. J. Rosseinsky, Nature 2019, 565, 213; e) S. Chen, S. Mukherjee, B. E. G. Lucier, Y. Guo, Y. T. Angel Wong, V. V. Terskikh, M. J. Zaworotko, Y. Huang, J. Am. Chem. Soc. 2019, 141, 14257; f) V. A. Milichko, S. V. Makarov, A. V. Yulin, A. V. Vinogradov, A. A. Krasilin, E. Ushakova, V. P. Dzyuba, E. Hey-Hawkins, E. A. Pidko, P. A. Belov, Adv. Mater. 2017, 29, 1606034; g) W. Danowski, T. van Leeuwen, S. Abdolahzadeh, D. Roke, W. R. Browne, S. J. Wezenberg, B. L. Feringa, Nat. Nanotechnol. 2019, 14, 488; h) C. S. Vogelsberg, F. J. Uribe-Romo, A. S. Lipton, S. Yang, K. N. Houk, S. Brown, M. A. Garcia-Garibay, Proc. Natl. Acad. Sci. U. S. A. 2017, 114, 13613.

[7] a) Y. V. Kaneti, J. Tang, R. R. Salunkhe, X. Jiang, A. Yu, K. C.-W. Wu, Y. Yamauchi, Adv. Mater. 2017, 29, 1604898; b) B. Y. Guan, X. Y. Yu, H. B. Wu, X. W. (D.) Lou, Adv. Mater. 2017, 29, 1703614; c) A. Indra, T. Song, U. Paik, Adv. Mater. 2018, 30, 1705146; d) M. H. Yap, K. L. Fow, G. Z. Chen, Green Energy Environ. 2017, 2, 218; e) R. V. Jagadeesh, K. Murugesan, A. S. Alshammari, H. Neumann, M.-M. Pohl, J. Radnik, M. Beller, Science 2017, 358, 326; f) L. R. Mingabudinova, A. S. Zalogina, A. Krasilin, M. I. Petrova, P. Trofimov, Y. A. Mezenov, E. Ubyivovk, P. Lönnecke, A. Nomine, J. Ghanbaja, T. Belmonte, V. A. Milichko, Nanoscale 2019, 11, 10155.

[8] a) I. Levchenko, K. Bazaka, O. Baranov, R. M. Sankaran, A. Nominé, T. Belmonte, S. Xu, Appl. Phys. Rev. 2018, 5, 021103; b) O. Baranov, K. Bazaka, H. Kersten, M. Keidar, U. Cvelbar, S. Xu, I. Levchenko, Appl. Phys. Rev. 2017, 4, 041302; c) D. Zhang, B. Gökce, S. Barcikowski, Chem. Rev. 2017, 117, 3990; d) S. V. Makarov, I. S. Sinev, V. A. Milichko, F. E. Komissarenko, D. A. Zuev, E. 
V. Ushakova, I. S. Mukhin, Y. F. Yu, A. I. Kuznetsov, P. A. Belov, I. V. Iorsh, A. N. Poddubny, A. K. Samusev, Yu. S. Kivshar, Nano Lett. 2018, 18, 535; e) X. Jiang, L. Zhang, S. Liu, Y. Zhang, Z. He, W. Li, F. Zhang, Y. Shi, W. Lü, Y. Li, Q. Wen, J. Li, J. Feng, S. Ruan, Y. J. Zeng, X. Zhu, Y. Lu, H. Zhang, Adv. Opt. Mater. 2018, 6, 1800561; f) X. Jiang, S. Liu, W. Liang, S. Luo, Z. He, Y. Ge, H. Wang, R. Cao, F. Zhang, Q. Wen, J. Li, Q. Bao, D. Fan, H. Zhang, Laser Photonics Rev. 2018, 12, 1700229; g) L. Lu, Z. Liang, L. Wu, Y. X. Chen, Y. Song, S. C. Dhanabalan, J. S. Ponraj, B. Dong, Y. Xiang, F. Xing, D. Fan, H. Zhang, Laser Photonics Rev. 2018, 12, 1700221; h) Y. Ge, Z. Zhu, Y. Xu, Y. Chen, S. Chen, Z. Liang, Y. Song, Y. Zou, H. Zeng, S. Xu, H. Zhang, D. Fan, Adv. Opt. Mater. 2018, 6, 1701166; i) L. Lu, X. Tang, R. Cao, L. Wu, Z. Li, G. Jing, B. Dong, S. Lu, Y. Li, Y. Xiang, J. Li, D. Fan, H. Zhang, Adv. Opt. Mater. 2017, 5, 1700301.

[9] a) R. N. Widmer, G. I. Lampronti, S. Anzellini, R. Gaillac, S. Farsang, C. Zhou, A. M. Belenguer, C. W. Wilson, H. Palmer, A. K. Kleppe, M. T. Wharmby, X. Yu, S. M. Cohen, S. G. Telfer, S. A. T. Redfern, F.-X. Coudert, S. G. MacLeod, T. D. Bennett, Nat. Mater. 2019, 18, 370; b) R. Gaillac, P. Pullumbi, K. A. Beyer, K. W. Chapman, D. A. Keen, T. D. Bennett, F.-X. Coudert, Nat. Mater. 2017, 16, 1149; c) T. D. Bennett, A. K. Cheetham, Acc. Chem. Res. 2014, 47, 1555; d) T. D. Bennett, S. Horike, Nat. Rev. Mater. 2018, 3, 431; e) D. A. Keen, T. D. Bennett, Phys. Chem. Chem. Phys. 2018, 20, 7857; f) L. Liu, Z. Chen, J. Wang, D. Zhang, Y. Zhu, S. Ling, K. W. Huang, Y. Belmabkhout, K. Adil, Y. Zhang, B. Slater, M. Eddaoudi, Y. Han, Nat. Chem. 2019, 11, 622; g) T. D. Bennett, A. K. Cheetham, A. H. Fuchs, F. X. Coudert, Nat. Chem. 2017, 9, 11.

[10] a) T. D. Bennett, Y. Yue, P. Li, A. Qiao, H. Tao, N. G. Greaves, T. Richards, G. I. Lampronti, S. A. T. Redfern, F. Blanc, O. K. Farha, J. T. Hupp, A. K. Cheetham, D. A. Keen, J. Am. Chem. Soc. 2016, 138, 3484; b) J. Hou, C. W. Ashling, S. M. Collins, A. Krajnc, C. Zhou, L. Longley, D. N. Johnstone, P. A. Chater, S. Li, M.-V. Coulet, P. L. Llewellyn, F.-X. Coudert, D. A. Keen, P. A. Midgley, G. Mali, V. Chen, T. D. Bennett, Nat. Commun. 2019, 10, 2580; c) T. D. Bennett, J.-C. Tan, Y. Yue, E. Baxter, C. Ducati, N. J. Terrill, H. H. M. Yeung, Z. Zhou, W. Chen, S. Henke, A. K. Cheetham, G. N. Greaves, Nat. Commun. 2015, 6, 8079.

[11] H. Jiang, S. Jin, C. Wang, R. Ma, Y. Song, M. Gao, X. Liu, A. Shen, G. J. Cheng, H. Deng, J. Am. Chem. Soc. 2019, 141, 5481.

[12] a) S. K. Sundaram, E. Mazur, Nat. Mater. 2002, 1, 217; b) G. Sciaini, M. Harb, S. G. Kruglik, T. Payer, C. T. Hebeisen, F.-J. Meyer zu Heringdorf, M. Yamaguchi, M. Horn-von Hoegen, R. Ernstorfer, R. J. D. Miller, Nature 2009, 458, 56; c) M. Harb, R. Ernstorfer, C. T. Hebeisen, G. Sciaini, W. Peng, T. Dartigalongue, M. A. Eriksson, M. G. Lagally, S. G. Kruglik, R. J. D. Miller, Phys. Rev. Lett. 2008, 100, 155504; d) P. B. Hillyard, K. J. Gaffney, A. M. Lindenberg, S. Engemann, R. A. Akre, J. Arthur, C. Blome, P. H. Bucksbaum, A. L. Cavalieri, A. Deb, R. W. Falcone, D. M. Fritz, Adv. Funct. Mater. 2020, 30, 1908292 www.advancedsciencenews.com www.afm-journal.de 1908292 (11 of 11) () 2019 WILEY-VCH Verlag GmbH \& Co. KGaA, Weinheim P. H. Fuoss, J. Hajdu, P. Krejcik, J. Larsson, S. H. Lee, D. A. Meyer, R. Pahl, D. A. Reis, J. Rudati, D. P. Siddons, K. Sokolowski-Tinten, D. von der Linde, J. B. Hastings, Phys. Rev. Lett. 2007, 98, 125501; e) A. Rousse, C. Rischel, S. Fourmaux, I. Uschmann, S. Sebban, G. Grillon, Ph. Balcou, E. Förster, J. P. Geindre, P. Audebert, J. C. Gauthier, D. Hulin, Nature 2001, 410, 65; f) C. W. Siders, A. Cavalleri, K. Sokolowski-Tinten, Cs. Tóth, T. Guo, M. Kammler, M. Horn von Hoegen, K. R. Wilson, D. von der Linde, C. P. J. Barty, Science 1999, 286, 1340; g) P. Saeta, J.-K. Wang, Y. Siegal, N. Bloembergen, E. Mazur, Phys. Rev. Lett. 1991, 67, 1023.

[13] M. O. Barsukova, S. A. Sapchenko, K. A. Kovalenko, D. G. Samsonenko, A. S. Potapov, D. N. Dybtsev, V. P. Fedin, New J. Chem. 2018, 42, 6408.

[14] a) N. Bréfuel, H. Watanabe, L. Toupet, J. Come, N. Matsumoto, E. Collet, K. Tanaka, J.-P. Tuchagues, Angew. Chem. 2009, 121, 9468; b) K. F. MacDonald, V. A. Fedotov, N. I. Zheludev, 
Appl. Phys. Lett. 2003, 82, 1087; c) E. Vauthey, ChemPhysChem 2012, 13, 2001; d) J. M. Crowley, J. Tahir-Kheli, W. A. Goddard, J. Phys. Chem. Lett. 2016, 7, 1198; e) P. Mori-Sanchez, A. J. Cohen, W. Yang, Phys. Rev. Lett. 2008, 100, 146401.

[15] a) A. I. Kuznetsov, A. E. Miroshnichenko, M. L. Brongersma, Y. S. Kivshar, B. Luk'yanchuk, Science 2016, 354, aag2472; b) V. A. Milichko, D. A. Zuev, D. G. Baranov, G. P. Zograf, K. Volodina, A. A. Krasilin, I. S. Mukhin, P. A. Dmitriev, V. V. Vinogradov, S. V. Makarov, P. A. Belov, Laser Photonics Rev. 2018, 12, 1700227; c) G. P. Zograf, M. I. Petrov, D. A. Zuev, P. A. Dmitriev, V. A. Milichko, S. V. Makarov, P. A. Belov, Nano Lett. 2017, 17, 2945; d) S. V. Makarov, M. I. Petrov, U. Zywietz, V. Milichko, D. Zuev, N. Lopanitsyna, A. Kuksin, I. Mukhin, G. Zograf, E. Ubyivovk, D. A. Smirnova, S. Starikov, B. N. Chichkov, Y. S. Kivshar, Nano Lett. 2017, 17, 3047; e) P. A. Dmitriev, D. G. Baranov, V. A. Milichko, S. V. Makarov, I. S. Mukhin, A. K. Samusev, A. E. Krasnok, P. A. Belov, Y. S. Kivshar, Nanoscale 2016, 8, 9721; f) C. Renaut, L. Lang, K. Frizyuk, M. Timofeeva, F. E. Komissarenko, I. S. Mukhin, D. Smirnova, F. Timpu, M. Petrov, Y. Kivshar, R. Grange, Nano Lett. 2019, 19, 877.

[16] a) V. V. N. Ravi Kishore, K. L. Narasimhan N. Periasamy, Phys. Chem. Chem. Phys. 2003, 5, 1386; b) Y. Lv, Y. Liu, X. Ye, G. Liu, X. Tao, CrystEngComm. 2015, 17, 526. 
a
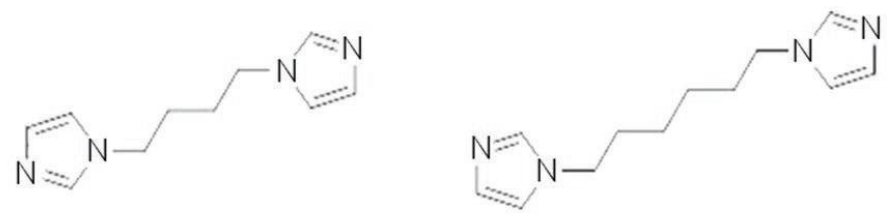

L4

L6

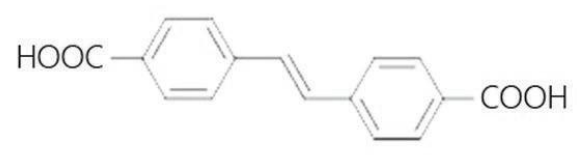

$\mathrm{H}_{2} \mathrm{sdc}$

b

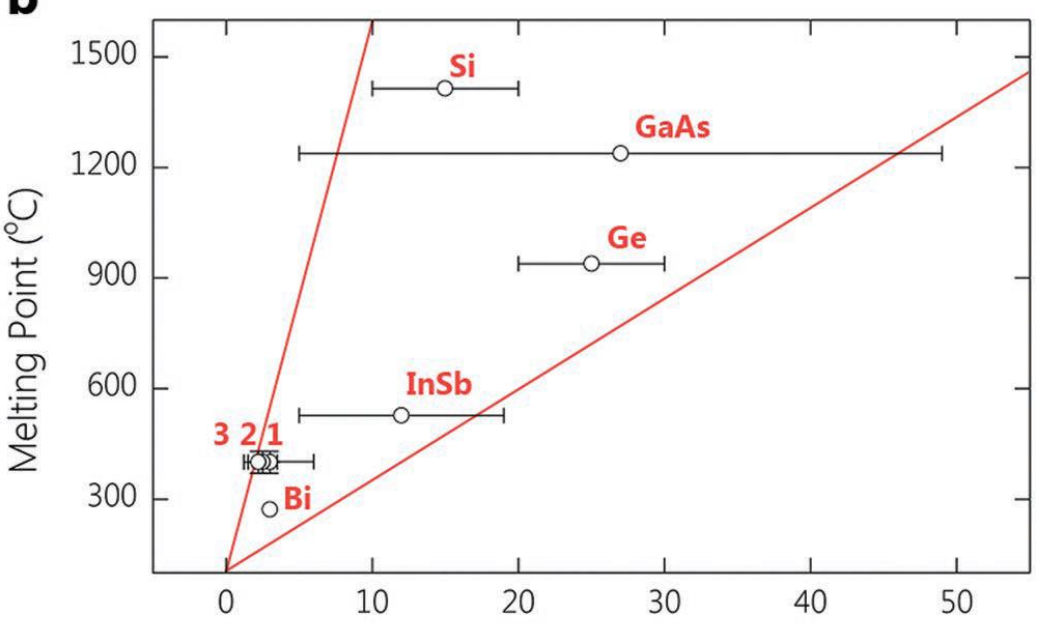

Fraction of excited electrons (\%)

Figure 1. a) Structural formulae of the ligands used to produce compounds 1-3. b) Fraction of electrons to be excited for ultrafast melting of crystalline semiconductors (Table S2, Supporting Information) versus temperature needed for classical melting with thermal equilibrium. 1, 2, and 3 present work, Bi,[12b] Si,[12c] InSb,[12d,e] Ge,[12f ] and GaAs.[12g] 

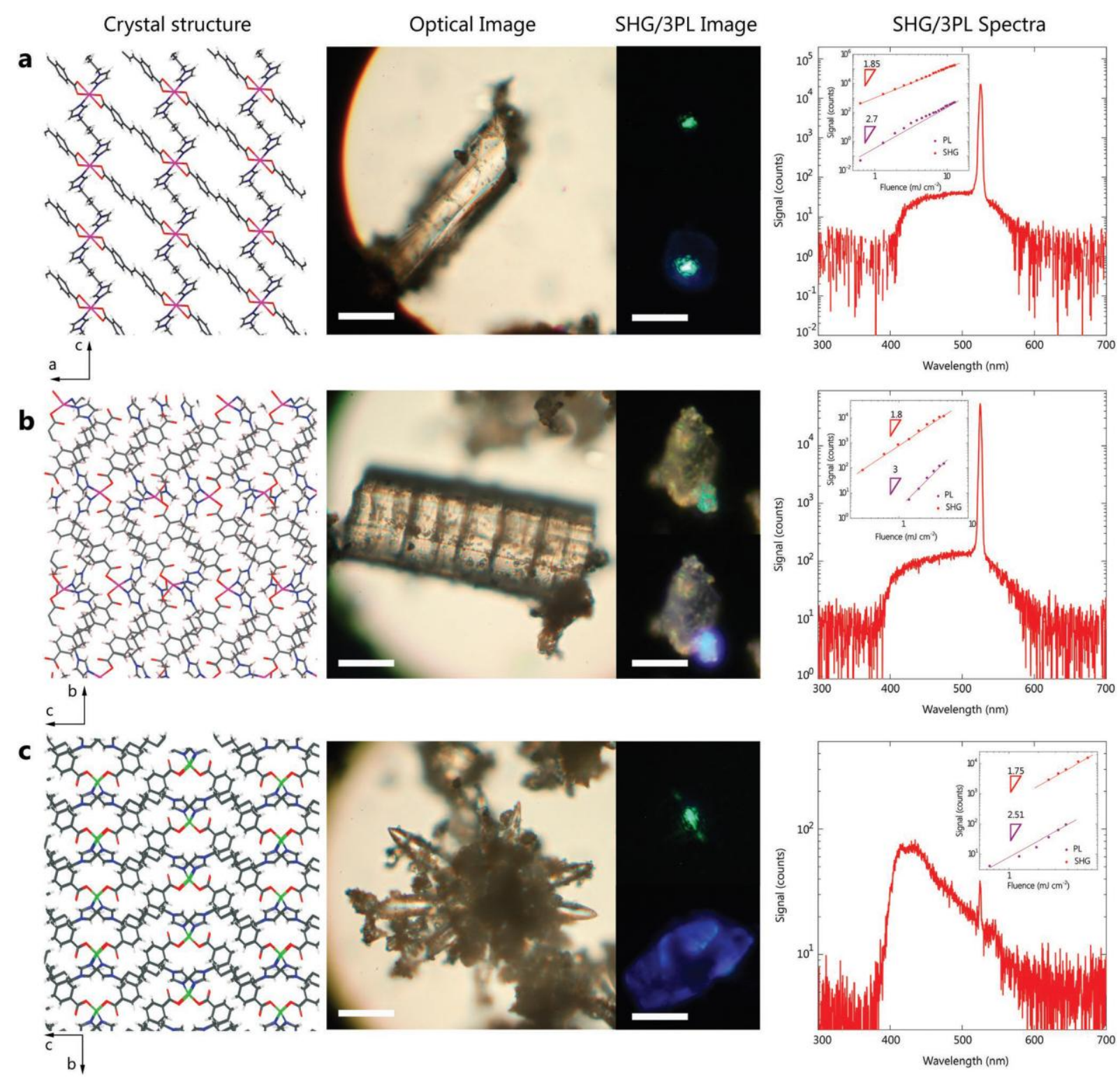

Figure 2. Characterization of initial MOFs. Crystal structure, optical image, SHG/3PL images as well as spectra of SHG/3PL with corresponding quadratic and cubic slope (inset) for a) compound $1, b$ ) compound 2, and c) compound 3. Scale bars, 10 and $5 \mu \mathrm{m}$ for optical and SHG/3PL images, respectively. 

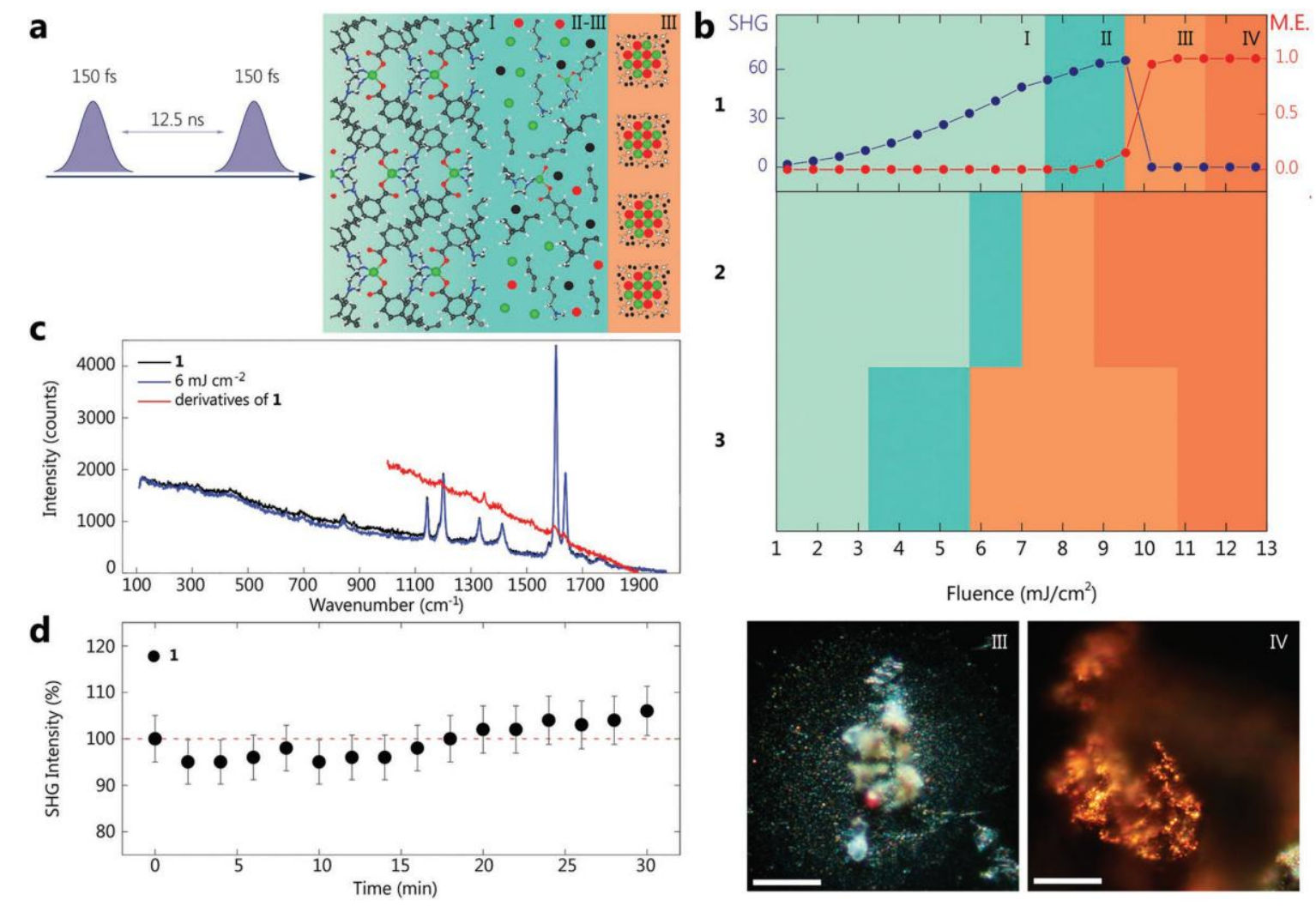

Figure 3. Ultrafast laser melting of MOFs. a) Scheme of interaction of MOF single crystal with $150 \mathrm{fs}$ $1050 \mathrm{~nm}$ laser pulses with $80 \mathrm{MHz}$ repetition rate. b) Four regimes (nonperturbed I, transition II, melting III, and burning IV regimes) of the interaction of MOFs with fs laser radiation with different fluence. As an example, the dependence of SHG intensity (in arbitrary units) and melting efficiency (M.E.) is shown for compound 1 demonstrating the step-like melting. Corresponding images of melted and burned single crystals are shown. Scale bar, $10 \mu \mathrm{m}$. For compounds 2 and 3 see Figure S8 in the Supporting Information. c) Raman scattering for single crystal of 1 before and after irradiation by $6 \mathrm{~mJ}$ $\mathrm{cm}^{-2}$, and for single derivatives of 1. d) Stability of SHG signal from single crystal of 1 irradiated by 6 $\mathrm{mJ} \mathrm{cm}{ }^{-2}$ for $30 \mathrm{~min}$. 

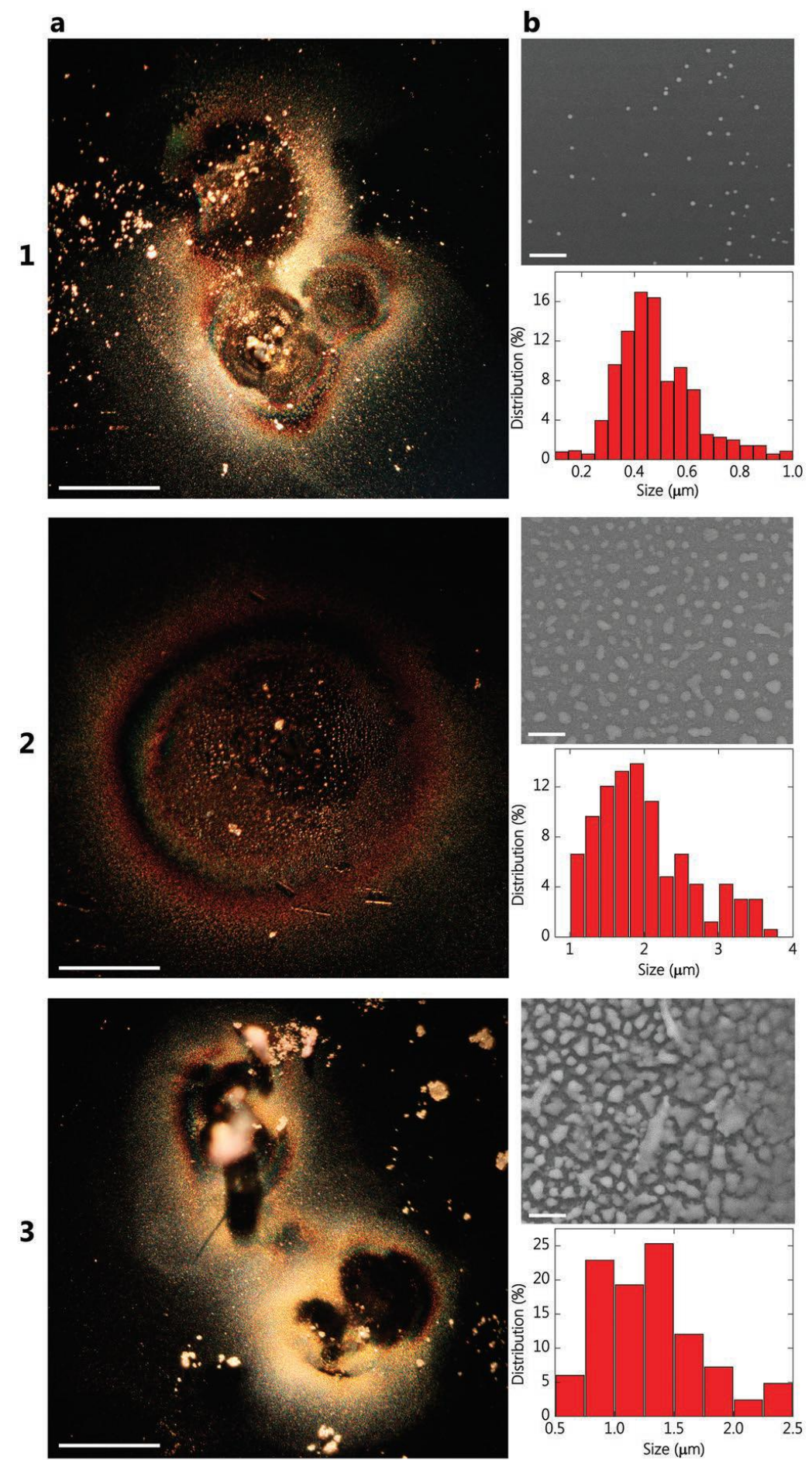

Figure 4. Morphology of MOF derivatives. a) Optical images and b) SEM micrographs and diameter distribution for corresponding derivatives obtained from compounds 1, 2, and 3. Scale bars: a) $50 \mu \mathrm{m}$ and b) $10 \mu \mathrm{m}$ for 1 and 2 , and $5 \mu \mathrm{m}$ for 3 . 


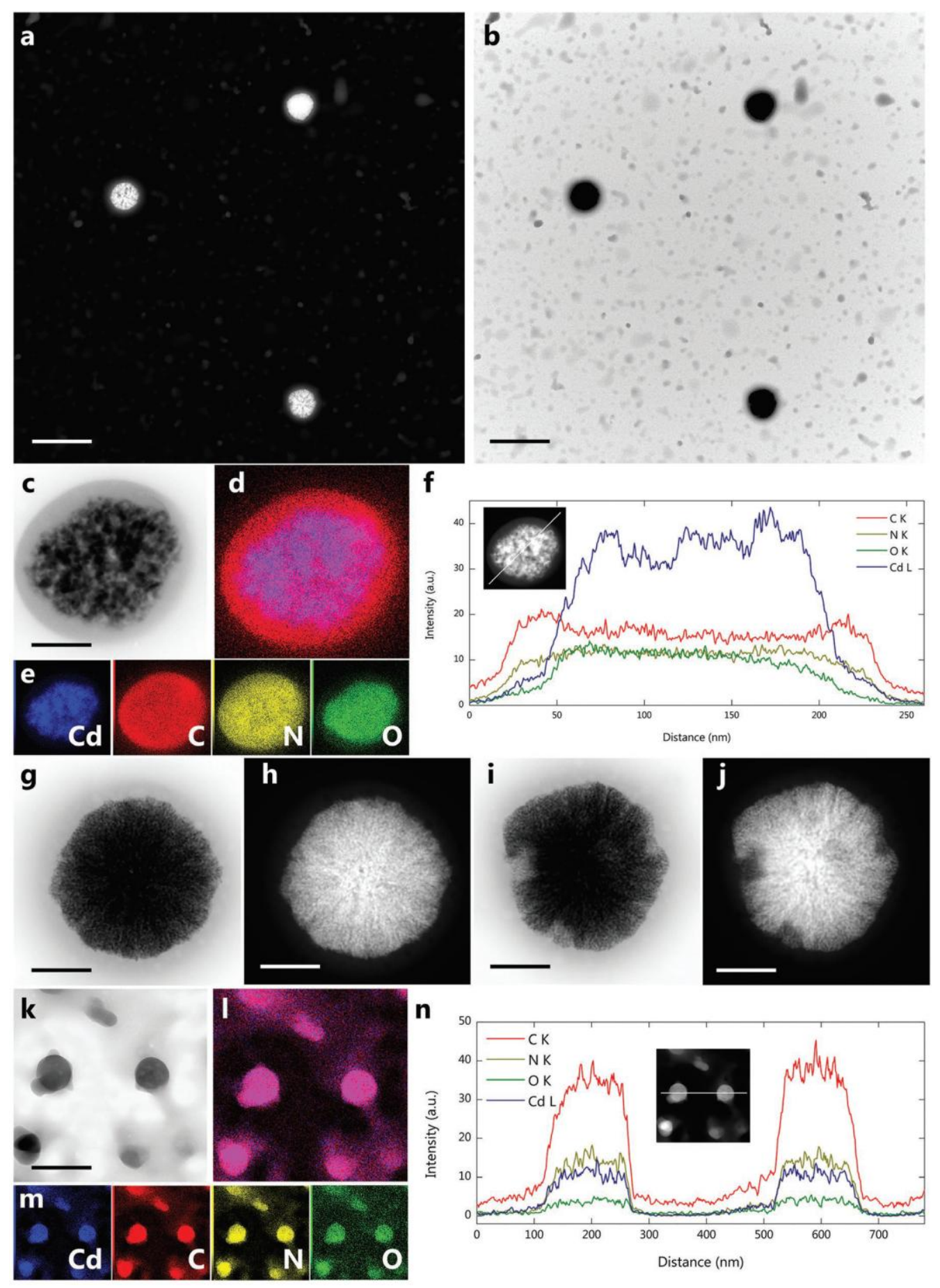

Figure 5. TEM analysis. a) HAADF (high angle annular dark field) and b) bright field TEM micrographs of MOF derivatives from compound 1. c,g-j) Three zoom in images of the core-shell derivatives. d-f) EDX analysis reveals the elemental ratio, and line scan confirms the core-shell structure. k) Bright field TEM image of the amorphous phase with $1, \mathrm{~m}$ ) corresponding elemental analysis and $n$ ) line scan representing the homogeneous element redistribution over the shape. Scale bars: a,b) $1 \mu \mathrm{m}$, c) $80 \mathrm{~nm}, \mathrm{~g}-\mathrm{j}) 200 \mathrm{~nm}$, and k) $200 \mathrm{~nm}$. 

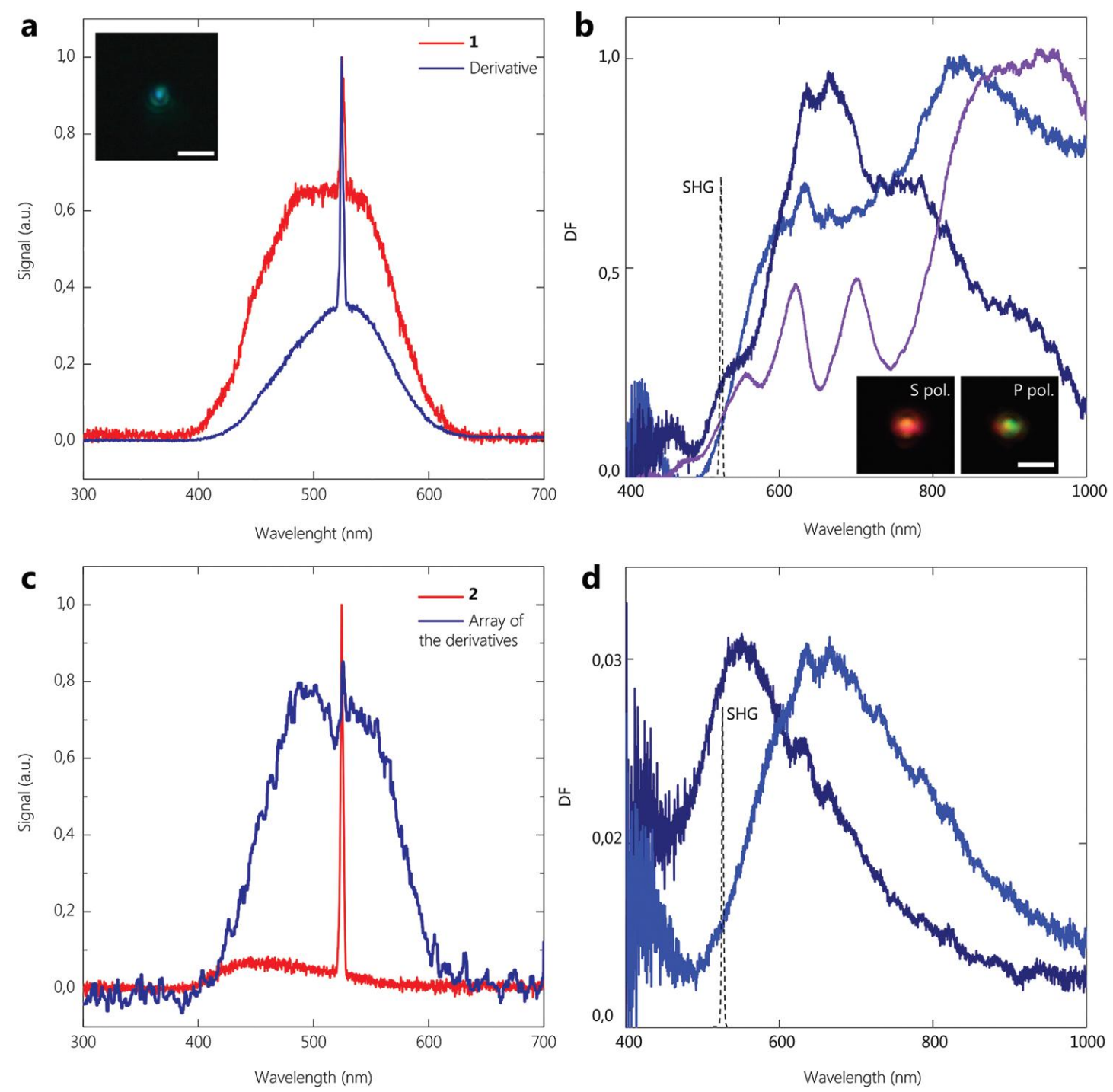

Figure 6. Optical properties of MOF derivatives. SHG and 3PL spectra from the derivatives of a) 1 and b) 2 normalized by maxima. Inset: Optical image of SHG-3PL signal from single derivative. Scale bar, $2 \mu \mathrm{m} . \mathrm{b}, \mathrm{d}$ ) Corresponding spectra of scattering of s polarized white light in dark-field geometry on single derivatives with diameter ranging from 500 to $800 \mathrm{~nm}$. The number of resonances (maxima of the curves) corresponds to intrinsic Mie-type modes generated inside the derivatives.[15] Inset: Optical image of derivative irradiated by s and p polarized light. Scale bar: $2 \mu \mathrm{m}$. 